\title{
Online CBT in the Treatment of Child and Adolescent Anxiety Disorders: Issues in the Development of BRAVE-ONLINE and Two Case Illustrations
}

\author{
Susan H. Spence and Caroline L. Donovan \\ Griffith University, Australia \\ Sonja March \\ University of Queensland, Australia \\ Amanda Gamble \\ Macquarie University, Australia \\ Renee Anderson and Samantha Prosser \\ University of Queensland, Australia
}

Amy Kercher

Macquarie University, Australia

Justin Kenardy

University of Queensland, Australia

\begin{abstract}
This paper describes the rationale for and development of an online cognitivebehavioural treatment for child and adolescent anxiety (BRAVE-ONLINE). It highlights the challenges involved in adapting a clinic-based intervention for delivery using the internet, with separate sessions for parents and their children (or adolescents). We outline strategies to ensure that young people remain engaged in online therapy, and describe techniques designed to optimize the alliance between clients and the online therapist. Two case studies are
\end{abstract}

Reprint requests to Susan Spence, Griffith University, Gold Coast Campus, Queensland 4222, Australia. E-mail: s.spence@griffith.edu.au 
presented that illustrate the practical and technical aspects of implementing the intervention, and demonstrate the feasibility of achieving successful outcomes using online delivery of CBT for child and adolescent anxiety. However, firm conclusions regarding the efficacy of this approach cannot be drawn until the results of randomized controlled trials are available. The paper identifies directions for future research.

Keywords: Anxiety, cognitive behaviour therapy, internet, online, computer.

\section{Introduction}

There is a good deal of evidence that cognitive behaviour therapy (CBT) is effective for a high proportion of children and adolescents with anxiety disorders (Hudson, 2005). It is worrisome therefore, that a significant proportion of anxious children and adolescents do not receive treatment (Essau, Conradt and Petermann, 2000). There are many factors that prevent young people from receiving therapy, such as the perceived stigma of attending a mental health clinic, poor access to mental health services, and time or financial constraints on the family (Booth et al., 2004).

The need to increase access to treatment is not unique to child and adolescent anxiety, and a variety of strategies have been developed to facilitate access to therapy for a wide range of physical and mental health problems. Technologies such as palm-top computers, email, the internet, DVDs, CD-ROMs, virtual reality, mobile phone text-messaging, and interactive voice mail systems have rapidly emerged as self-help treatments or adjuncts to therapistguided interventions for mental health problems. These technologies increase accessibility, convenience and flexibility of treatment and, if effective, may be more cost-effective than face-to-face therapy (Griffiths and Christensen, 2006; Kaltenthaler et al., 2006). Cognitive behavioural therapy may be particularly suitable for computer-based administration because of its structured and systematic format (Kenardy and Adams, 1993).

\section{The effectiveness of computer-based treatments with anxious adults}

There are now several published studies in the adult literature demonstrating effective use of computer-based CBT treatment for anxiety disorders either as a stand-alone treatment or as an adjunct to traditional face-to-face therapy (Kenardy et al., 2003; Spek et al., 2007). Generally, the results suggest that although self-help, computer-based therapy for anxious adults produces significant reductions in anxiety, these effects are relatively small and are more substantial if computer delivery of treatment is accompanied by regular contact with a therapist either by phone, email, or face-to-face (for a review see Spek et al., 2007).

\section{Effectiveness of computer-based CBT with anxious children and adolescents}

Despite the recent flurry of interest in the use of computer-based technologies for adult anxiety disorders, substantially less attention has focused on the development and evaluation of computer-based treatment of anxiety disorders with children and adolescents. Positive outcomes have been reported in case studies involving spider phobia (Nelissen, Muris and Merckelbach, 1995) and selective mutism (Fung, Manassis, Kenny and Fiksenbaum, 2002), and a small, randomized controlled trial of the treatment of spider phobic children (Dewis 
et al., 2001). Recently, Spence, Holmes, March et al. (2006) reported a randomized controlled trial that compared clinic-delivered CBT for the treatment of anxiety disorders in children aged 7-12 years, with the same intervention in which half of the sessions were presented over the internet and half in the clinic. Results indicated that both conditions produced significant reductions in anxiety problems when compared to no-intervention, and that there were minimal differences in outcome between the two treatments. This study demonstrated the feasibility of translating at least some parent and child CBT sessions for child anxiety into a format delivered over the internet, with minimal reduction in therapeutic outcome.

Yet to be determined, however, is whether a CBT approach to the treatment of child and adolescent anxiety can be adapted for full internet delivery without clinical attendance. Given the encouraging results for computer-based and computer-assisted treatments for anxiety in adults, and the high level of accessibility and acceptability of internet-delivered information to children and adolescents, there is a strong case to be made for the development and evaluation of internet-delivered CBT for child and adolescent anxiety disorders.

The present paper describes the development of a CBT program for child and adolescent anxiety disorders that is delivered entirely via the internet, with a relatively small level of therapist support. The program is being evaluated in a series of randomized clinical trials over the next 2 years. In this paper, we highlight the challenges involved in adapting a clinicbased intervention for delivery using the internet, with separate sessions for parents and their children (or adolescents). Specifically, we outline strategies to ensure that young people remain engaged and interested in online therapy, and describe techniques to optimize the therapeutic alliance between the young person and their online therapist. Two case studies are presented that illustrate the practical and technical aspects of the intervention, and some of the difficulties that may arise. Finally, we propose some important directions for future research.

\section{Method}

\section{Content of the intervention}

The intervention (BRAVE-ONLINE) was adapted from a clinic-based CBT anxiety treatment program for children aged 7-14 years (Spence, Holmes, March et al. 2006). The BRAVE Program is based on theoretical and empirical research relating to the psychosocial determinants of child anxiety (e.g. Dadds, Davey and Field, 2001; Ollendick, King and Muris, 2002; Rapee and Spence, 2004; Silverman and Treffers, 2001; Vasey and Dadds, 2001) and evidence-based, cognitive-behavioural interventions for childhood anxiety (Barrett, 1998; Kendall, 1994; Rapee, Wignall, Hudson and Schniering, 2000; Spence, Donovan and Brechman-Toussaint, 2000). An evaluation of clinic-based delivery of the BRAVE program indicated significant reductions in anxiety symptoms and disorders, consistent with those evident elsewhere in the literature (Spence, Holmes, March et al., 2006).

Both clinic and internet versions of the BRAVE Program are universal anxiety treatment programs designed to treat Social Phobia, Generalised Anxiety Disorder, Separation Anxiety Disorder and Specific Phobia. Children with Panic Disorder, Post Traumatic Stress Disorder and Obsessive Compulsive Disorder are not included in the programs as we feel that they need more specialized content. For individuals with multiple anxiety diagnoses, treatment focuses on the anxiety disorder identified as primary (i.e. most severe), according to clinician severity ratings (CSR) on the pre diagnostic interview. There are two versions of the program; one for 
children aged 8 to 12 years (Spence, March and Holmes, 2005) and one for teenagers aged 13-17 years (Spence, Holmes, Donovan and Kenardy, 2006). These versions take into account developmental differences in cognition, emotion and behaviour, and also the reading level of the young person and age-appropriateness of examples and content.

Consistent with the original clinic version, BRAVE - ONLINE involves 10 weekly child/adolescent sessions, each taking approximately 60-minutes to complete, followed by two booster sessions, 1 month and 3 months after completion of the initial program. Parents complete six sessions in the child program, and five slightly longer sessions in the teenage program.

The anxiety management strategies covered include recognition of the physiological symptoms of anxiety, relaxation strategies (progressive muscle relaxation, guided imagery, and deep breathing), cognitive strategies of coping self-talk and cognitive restructuring, graded exposure, problem solving techniques, and self-reinforcement of "brave" behaviour. Participants consolidate learning of these strategies through completion of weekly homework tasks, known as "extreme challenges".

Parent sessions focus on psychoeducation about child anxiety, contingency management, relaxation training, and information about cognitive restructuring, graded exposure and problem solving.

\section{Adapting the program for internet delivery}

Families that meet inclusion criteria for the program are introduced to their internet therapist during a 15-minute telephone call that also summarizes what will be involved in the treatment. Subsequent contact with the online therapist is limited to a mid-program phone call (described later) and brief, weekly email contacts in which the therapist provides feedback to the parent and child about the activities and homework completed during the online session. Each therapist keeps a record of the length of all contacts. Currently, we estimate that the online therapist spends about 10 to 15 minutes per family per week in reviewing data from sessions, client homework assignments, and preparing email responses. Given that each session normally takes around 60-90 mins to conduct in the clinic, this is a considerable saving of therapist time.

The resource folder. Before commencing the program, participants are provided with a folder that includes the website address, username information, program and session outlines, a tutorial guide to demonstrate how the program is used, BRAVE staff contact details, a relaxation $\mathrm{CD}$, and handouts. Participants are asked to use this folder to store handouts that are printed during each session, homework sheets, and information regarding their exposure hierarchy.

Engaging participants. One of our major challenges is to ensure that the young person attends to and processes the information provided in the absence of a face-to-face therapist who would normally check for attention and understanding. One specific way we have attempted to capture the attention of young people is to make the content of BRAVE - ONLINE visually appealing and interesting. Thus, we use a minimal amount of text per page, and incorporate bright, eye-catching graphics including cartoon animation and, for the teenage program, reallife pictures. The program also uses "quizzes" and interactive tasks to facilitate attention and comprehension of material (see Figure 1). Each session begins with a recap and quiz 


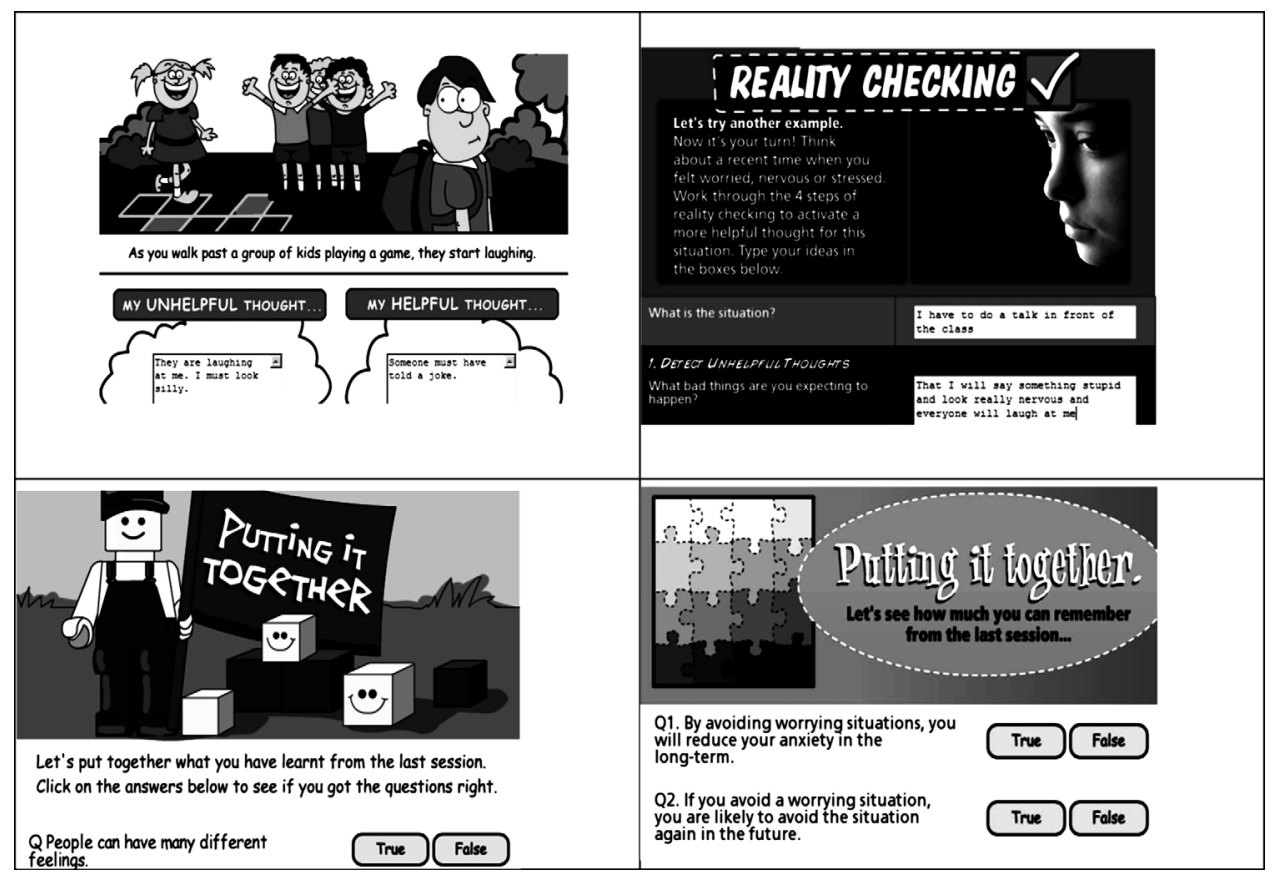

Figure 1. Use visually appealing and interactive activities for the child and teenage programs

relating to the previous session, and ends with a summary and quiz relating to the current session. The young person is provided with automated feedback regarding the accuracy of their responses via pop-ups, to ensure their understanding of key concepts. Interactive games are another technique used to present and consolidate program information in an interesting way.

Age-related considerations. Although the content is consistent across the child and teenage programs, the presentation differs to account for developmental factors. The content of the child program was designed for children with a literacy level of age 8, whereas the adolescent program was aimed at a minimum reading level of age 12. Accordingly, the teenage program involves more complex text, examples and stories, has more advanced graphics, and is interspersed with a greater number of interactive exercises.

One of the techniques used to teach coping skills involves the use of cartoon and reallife characters to illustrate the use of CBT strategies. With younger children, we drew on research relating to hero characters (Cornwall, Spence and Schotte, 1996) and developed "Brave Buddy" as a cartoon coping model. In contrast, we drew on research relating to the effectiveness of modelling by peers as a strategy for teaching new skills and knowledge for the teenage program (Bandura, 1977). This involves four young people, each of whom experiences one of the four main anxiety disorders that the program is designed to treat. Both Brave Buddy and the teenage characters provide "models" for young people in the use of coping strategies to overcome anxiety problems. 

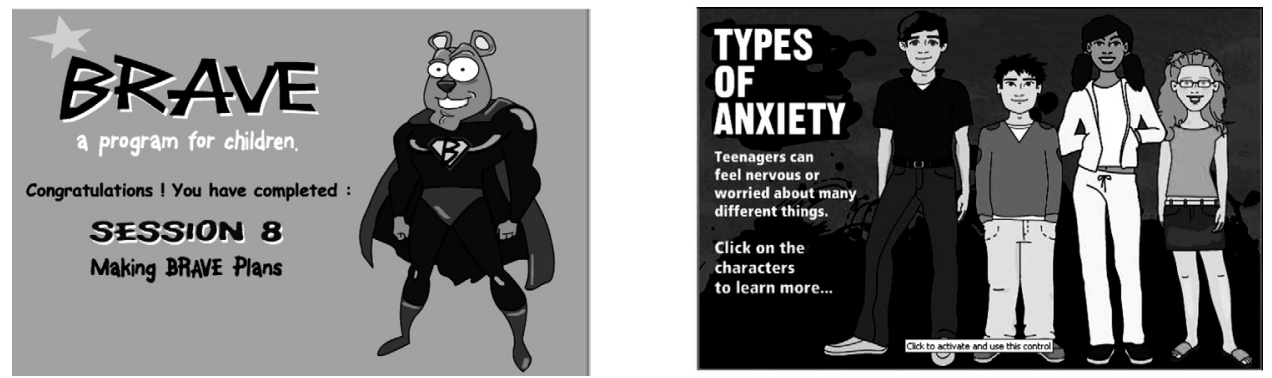

Figure 2. The use of characters to illustrate techniques for managing anxiety
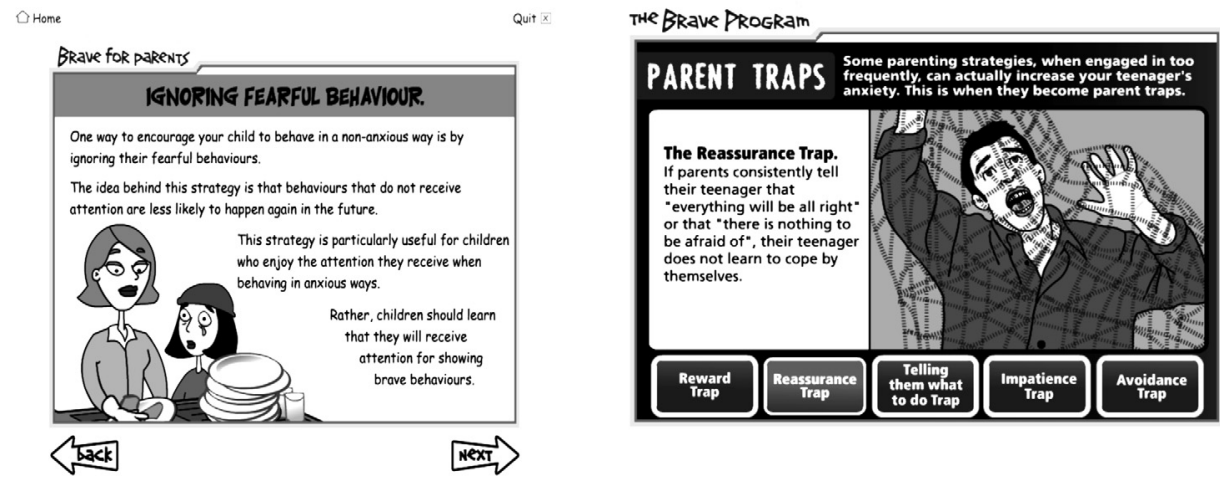

Figure 3. Examples of content presentation in the parent program

The parent program. The parent program corresponds closely to the child and adolescent programs; however, fonts and graphics were changed to incorporate parent characters and to include adult-level language.

\section{Maximizing the therapeutic alliance: creating a virtual therapist}

The quality of the therapeutic alliance has consistently been found to predict more effective psychological treatment outcome and to be associated with higher levels of treatment compliance and lower levels of treatment drop-out (see Howgego, Yellowlees, Owen, Meldrum and Dark, 2003; Martin, Garske and Davis, 2000 for reviews). In order to optimize the quality of the therapist-client relationship, a series of strategies are used. First, given that one of the aims of developing an internet program was to reduce concerns about stigma and acceptability of treatment to young people, we avoid the use of the term "therapist" and use the concept of "trainer" instead. Each participant is assigned their own "BRAVE trainer" who they are introduced to on a webpage that includes a photograph and short biography. Participants complete an online exercise in which the young person and therapist provide each other with appropriate information about themselves through a series of guided questions, to produce a sense of "knowing" each other. 
Automated, computer-generated weekly emails, both before and after each session, are also used to enhance the therapeutic alliance. Upon completion of a session, parents and young people are sent an automatic email from their BRAVE trainer congratulating them on finishing their session. Seven days later another automatic email is sent from their BRAVE trainer informing the participants that their next session is ready for completion. Automatic emails use the participants' first name, refer to them personally, and are sent on behalf of the client's particular BRAVE trainer.

In addition, the therapist monitors participants' responses and provides written feedback by email. The computer stores information about responses to tasks and homework assignments, and the therapist is able to examine the responses at a convenient time each week and generate personalized email feedback to reinforce effort and success and provide corrective information if required.

The program also uses visual "pop-ups" to provide instant feedback about responses to quizzes and session tasks, as well as giving praise and encouragement. Furthermore, the popups provide an explanation regarding correct and incorrect answers. In the teenage program, these pop-ups are personalized, with both the participants' name and the name of the BRAVE trainer inserted into the computer-generated response.

Developing the exposure hierarchy. For most CBT strategies, transformation of content from the clinic to internet medium was reasonably straightforward. However, the graded exposure component of the program was significantly more difficult. We found it necessary to include a therapist telephone call following the internet session that focused on development of the young person's hierarchy. In the child program, development of the exposure hierarchy involves considerable parental input and relies heavily on the therapist 30-minute phone call for guidance. For the teenage program, hierarchy development takes place over two internet sessions, allowing the teenager to develop their own hierarchy without parental input and with less assistance from the therapist during the phone call. The exposure hierarchy focuses on situations relevant to the primary anxiety diagnosis.

\section{Case illustrations}

In order to illustrate the issues involved in the online implementation of the program, two case studies are presented. Although both cases experienced positive outcomes from the program, they are not presented as indicators of the effectiveness of the intervention. Rather, they are used to highlight the complexities and challenges involved with this type of treatment delivery.

\section{Measures}

Diagnostic status. Diagnostic status was assessed using the Anxiety Disorders Interview Schedule, Child and Parent Versions (ADIS-C/P; Silverman and Albano, 1996), administered over the telephone. A combined ADIS-C/P diagnosis that took into account both the parent and child responses to interview questions was used, supplemented by clinical severity rating (CSR) ranging from $0-8$ that was provided by the interviewer. The telephone interview version of the ADIS-C/P has been found to have high inter-rater reliability (Lyneham and Rapee, 2005). The ADIS-C/P and all outcome questionnaires were administered at pre-treatment, post-treatment, and 6-month follow-up by an interviewer who was blind to experimental condition. 
Children's Global Assessment Scale. The Children's Global Assessment Scale (CGAS; Schaffer et al., 1983) was used to assess global level of functioning. Based on information provided in the ADIS-C/P interviews, the interviewer gave a rating of $0-100$ on the CGAS scale. According to Schaffer et al. (1983), scores of 81-100 on the CGAS indicate a normal level of functioning, scores of 61-80 represent slight disability, scores of 41-60 indicate moderate disability, and scores of 1-40 represent serious disability.

Spence Child Anxiety Scale. Child anxiety was measured through child- and parent-report versions of the Spence Children's Anxiety Scale (SCAS; Spence, 1998). Both the childreport version of the SCAS (SCAS-C) and the parent-report version (SCAS-P) comprise 6 subscales relating to social phobia, separation anxiety, panic-agoraphobia, obsessive compulsive disorder, generalized anxiety and fear of physical injury, plus a total score. The SCAS-C and SCAS-P have demonstrated sound psychometric properties (Muris, Schmidt and Merckelbach, 2000; Spence, Barrett and Turner, 2003).

Child Behaviour Checklist. Child internalizing behaviour was assessed by the internalizing subscale of the Child Behaviour Checklist (CBCL; Achenbach and Rescorla, 2001). The CBCL is a 113-item parent-report questionnaire, comprising internalizing and externalizing behaviour subscales. Only the scores on the internalizing subscale are presented here. The psychometric properties of the CBCL are well established (Achenbach and Rescorla, 2001).

Youth Self Report (YSR; Achenbach and Rescorla, 2001). The YSR is a 112-item youthreport questionnaire, comprising internalizing and externalizing behaviour subscales. This scale was completed only by the adolescent participants, and only the scores on the internalizing subscale are reported here. The psychometric properties of the YSR are well established (Achenbach and Rescorla, 2001).

Self efficacy. After session 1, parents and children completed a brief self efficacy scale, designed to examine perceived competence in relation to the skills required to use the BRAVEONLINE treatment program. The scale consists of 10 items based on Bandura's (1977) theory of self efficacy. Children and parents rate on an 11-point Likert scale, from 0 to 10, the extent to which they are confident in their ability to use the online program, correspond with their brave trainer via email and phone, and implement the skills learned in the program. The total score has a possible range of 0 to 100, with higher scores indicating greater self efficacy.

Treatment outcome expectancy. Parents and children completed a 5-item scale designed to measure their expectations about treatment outcome and treatment credibility after Session 1. Items are based on Borkovec and Nau's (1972) conceptualization of treatment credibility and expectancy, and assess clients' expectations about the program's ability to help them overcome anxiety, as well as their own ability to complete the program and use the skills learned during the program to master their anxiety. Responses are rated on a 10-point Likert scale, from 0 (not at all) to 9 (completely). The total score has a possible range of 0 to 45 , with higher scores indicating greater expectancy and credibility ratings of treatment outcome.

Working Alliance Inventory - Short Form. The quality of the therapeutic alliance was assessed using the 12-item Working Alliance Inventory-Short Form (WAI-S: Tracey and Kokotovic, 1989). Minor changes were made to the wording of the child version of the WAI-S so that it could be more easily understood by the younger age group. The total score has a possible range of 12 to 84 , with higher scores being indicative of stronger therapeutic alliance. 
The WAI-S displays excellent internal reliability (Tracey and Kokotovic, 1989). The WAI-S was completed after Session 3 and at post-treatment.

Treatment satisfaction. Immediately following the end of treatment sessions, satisfaction with the intervention was assessed by an 8-item questionnaire. Parent and child rated on a 6-point scale from 0 (not at all) to 5 (very much) how satisfied they were with various aspects of the treatment program. The resulting total score has a possible range from 0 to 40 , where higher scores are indicative of a higher level of satisfaction with treatment. In addition, three open-ended questions provide information on what the clients liked most about the program, what they liked least, and which parts of the program they perceived to be most important components of therapy.

\section{Assessment of change}

Several methods were used to assess the level of clinical improvement in the case studies. First, changes in diagnostic status were used based on the clinical interviews. We also examined baseline scores on symptom questionnaires to determine whether scores were initially in the clinical range and whether these had shifted into the non-clinical range at post-treatment. T-scores were used to determine clinical status on the CBCL and YSR internalizing subscales, and a score of $1 \mathrm{SD}$ above the mean for the relevant age and gender group was used to determine clinical status for total scores and subscale scores on the SCAS-C and SCAS-P using norms from Spence (1998) and Nauta et al. (2004). The reliable change index (Jacobson and Truax, 1991) was calculated to determine whether the magnitude of the change was statistically reliable. As exact test-retest reliability data for SCAS-P were not available, the test-retest reliability was estimated as. 70 for the 12 week period in calculating the standard error of the difference. The standard error of difference scores were then multiplied by 1.64 to determine the level of reliable change required for $p<.05$ (two-tailed) (Iverson, 2001).

\section{Case Study 1: Method}

\section{Participant}

Presenting problem. CF was a 10-year old Caucasian boy who was an only child, living with his biological mother. His primary presenting problems related to anxiety about separating from his mother, and excessive worry about his own safety and that of his mother. These fears were reflected in behaviours including refusal to attend after-school activities (e.g. sport) unless his mother remained in sight, refusing to visit or spend the night at friends' houses without his mother, and sleeping in his mother's bed on a nightly basis. CF also presented with a significant fear of the dark and worried excessively about a range of topics including schoolwork, being "perfect", social interactions, and current/world affairs including terrorism and crime.

History of presenting problem and previous assistance. CF's parents separated prior to his birth and he did not have contact with his father. He demonstrated a long history of anxiety, with difficulty in separating from primary caregivers and engaging in independent activities evident from 2-3 years of age. His mother reported that he had slept in her bed since he was approximately 4 years old and that she had made several attempts to encourage him to sleep 
in his own bed that resulted in only minor, short-term improvements. At age 7, CF attended 4 sessions with a psychologist for assistance in anxiety management; however, only minimal, short-term reductions in anxiety resulted. There was no family history of anxiety or other psychiatric difficulties.

\section{Results}

\section{Baseline assessment}

Table 1 outlines diagnostic severity ratings and questionnaire results for $\mathrm{CF}$ at pre-treatment, post-treatment, and 6-month follow-up. The ADIS-C/P indicated that CF met DSM-IV criteria for separation anxiety disorder (SAD), specific phobia (darkness), and generalized anxiety disorder (GAD) at pre-treatment. The CSR and CGAS ratings placed him within the clinical range at pre-assessment. Scores on the parent- and child-report measures were consistent with the ADIS-C/P diagnoses, with particularly high scores being noted on the SAD, GAD, physical injury, and OCD subscales and total anxiety score of the SCAS-P. Similarly, the internalizing sub-scale scores and total internalizing score of the CBCL were in the clinical range. No significant elevations were noted on the child SCAS-C report prior to treatment, although CF's score on the separation anxiety subscale approached the clinical range.

\section{Treatment participation}

Session and activity completion. Both CF and his mother actively participated in treatment, completing all child and parent sessions at regular, weekly intervals, with an average of 30 minutes spent on each session. Treatment compliance was high for CF and his mother, with approximately $95 \%$ of activities throughout the program being completed. With the exception of some initial resistance towards practising the relaxation exercises, CF and his mother appeared to attain a good understanding of the anxiety management strategies and, based on session responses, were able to effectively apply the techniques to personal anxiety-provoking situations.

Graded exposure tasks. CF's fear of sleeping in his own bed was the focus of graded exposure. With the assistance of a 30-minute phone-call, in addition to internet instructions, $\mathrm{CF}$ and his mother worked together to construct the exposure hierarchy. In the remaining sessions, the online feedback indicated that $\mathrm{CF}$ and his mother worked through all steps of the hierarchy, practising tasks most nights and moving up the hierarchy at the rate of one step per week. Both reported success with the technique, indicating that they had achieved the goal of CF being able to sleep in his own bed on most nights.

Generalizing their skill in hierarchy construction, $\mathrm{CF}$ and his mother independently created and worked through an additional exposure hierarchy aimed at reducing CF's anxiety about being picked up from school by his grandmother. Although therapist assistance was not provided with this particular exposure hierarchy, reports from CF's mother indicated that he also progressed well through the steps of this hierarchy.

Self efficacy and treatment outcome expectancy. CF and his mother reported high levels of self-efficacy regarding their capacity to complete the BRAVE - ONLINE treatment program (CF: 88/100; Mother: 100/100). CF and his mother also reported moderate to high levels of 
Table 1. Case Study 1- Pre- to post-treatment and follow-up outcome scores and diagnostic information

\begin{tabular}{|c|c|c|c|}
\hline Measure & Pre & Post & 6-month follow-up \\
\hline CSR SAD (Primary) & 7 & 4 & nil \\
\hline CSR Specific Phobia & 5 & 5 & nil \\
\hline CSR GAD & 5 & nil & nil \\
\hline Combined CGAS & $50^{\# \#}$ & 69 & 74 \\
\hline \multicolumn{4}{|l|}{ SCAS-C } \\
\hline OCD & 2 & 1 & 0 \\
\hline Social phobia & 3 & 3 & 4 \\
\hline Panic & 1 & 1 & 1 \\
\hline Separation & 6 & 6 & $9^{\# \#}$ \\
\hline Physical injury & 3 & 3 & 5 \\
\hline GAD & 5 & 7 & 6 \\
\hline Total & 20 & 21 & 25 \\
\hline \multicolumn{4}{|l|}{ SCAS-P } \\
\hline OCD & $7^{\# \#}$ & 1 & $0^{*}$ \\
\hline Social phobia & 6 & 3 & 4 \\
\hline Panic & 1 & 0 & 1 \\
\hline Separation & $14^{\# \#}$ & $8^{\# \#}$ & $7^{\# \# *}$ \\
\hline Physical injury & $7^{\# \#}$ & 5 & 4 \\
\hline GAD & $5^{\# \#}$ & 4 & $2^{*}$ \\
\hline Total & $40^{\# \#}$ & 21 & $17^{*}$ \\
\hline \multirow[t]{2}{*}{ CBCL - Internalizing } & $18^{\# \#}$ & 4 & 3 \\
\hline & $\mathrm{T}=71^{\# \#}$ & $\mathrm{~T}=49$ & $\mathrm{~T}=46^{*}$ \\
\hline Satisfaction - Parent & - & 35 & - \\
\hline Satisfaction - Child & - & 37 & - \\
\hline WAI & Session 3 & & \\
\hline Child report & 71 & 80 & \\
\hline Parent report & 83 & 77 & \\
\hline
\end{tabular}

CSR = Clinician Severity Rating; CGAS = Children's Global Assessment Scale; SCAS-C = Spence Children's Anxiety Scale Child Version; SCAS-P = Spence Children's Anxiety Scale - Parent Version; $\mathrm{CBCL}=$ Child Behaviour Checklist; WAI-S = Working Alliance Inventory

\#\# clinical or elevated scores, ${ }^{*} \mathrm{RCI}$ values indicate a reliable change at the $5 \%$ significance level or better.

expectancy with regard to treatment outcome and the program's ability to help them overcome anxiety (CF: 29/45; Mother: 31/45).

Therapeutic alliance and client satisfaction. $\mathrm{CF}$ and his mother reported a strong therapeutic alliance with their BRAVE trainer, as shown in the WAI-S scores in Table 1, and high levels of satisfaction with the program following treatment. The exposure hierarchy, problem solving and thought challenging activities were identified as the most important aspects of the program. CF and his mother both reported that they liked the self-directed nature of the program because they were able to complete sessions in their own time and at their own pace. 


\section{Post-treatment assessment}

At post-treatment, the clinical interviews and questionnaire data indicated that although $\mathrm{CF}$ was showing some marked reductions in anxiety symptoms, he continued to demonstrate clinical levels of both SAD and specific phobia. However, by 6-month follow-up he no longer held a DSM-IV diagnosis of any anxiety disorder and the CGAS rating was well within the normal range. Although he occasionally slept in his mother's bed, he no longer reported any other separation difficulties. At 6-month follow-up, CF was able to engage in activities he had previously been unable to do, such as going on school camp and spending nights away from his mother.

Maternal responses on the internalizing sub-scale of the CBCL demonstrated reductions into the non-clinical range at post-treatment, with improvement being maintained at 6-month follow-up. Scores on the SCAS-P showed similar improvements. CF's own ratings on the SCAS-C did not reflect these gains, but he may have under-reported his anxiety symptoms at pre-treatment. Indeed, CF was also reluctant to report symptoms of anxiety during the clinical interview, a characteristic that has been noted in anxious children elsewhere in the literature (Silverman, 1994).

Reliable Change Indices (RCI; Jacobson and Truax, 1991) were calculated for the pretreatment to 6-month follow-up difference for the SCAS-C, SCAS-P and CBCL scores. Clinically reliable change was shown for scores on the OCD, SAD, and GAD subscales, and total score of the SCAS-P, and the internalizing subscale of the CBCL at a criterion of $p<.05$ or better. A clinically reliable change was not found for child report on the SCAS-C, but these scores were not in the clinical range initially.

Taken together, the outcomes for this case were generally positive and provide an illustration of the way in which an online CBT program can be used successfully in the treatment of child anxiety. Discussion of the case and its outcomes will be presented following the results of the second case study.

\section{Case Study 2: Method}

\section{Participant}

Presenting problem. AR was a 17-year-old Caucasian female, living at home with her biological mother and father. She had recently commenced study at music college. The parents were both employed in a small family business. AR was referred to the program by her mother who was concerned that her daughter experienced high levels of anxiety regarding social situations. Her mother reported AR to be shy and withdrawn in situations where she was required to interact with others, or where she might be the focus of attention. AR reported having fewer friends than other people her age and worried about "fitting in". She avoided going to parties and dances, and found it difficult to initiate and maintain conversations with others, particularly unfamiliar people and female peers. Anxiety in social situations had a significant impact on AR's social functioning and had prevented her from achieving several goals. Although a talented pianist and dancer, her fear of negative evaluation prevented her from auditioning for parts in musicals and bands. She also avoided applying for a position as music tutor because the application process involved playing the piano in front of an interview panel. She found it particularly difficult to speak in tutorials at college. 
In addition to her social concerns, AR experienced excessive and uncontrollable worry about a range of situations including her grades, her performance on tasks such as dancing and playing musical instruments, and her relationship with her father, whom she described as being easily angered. She also worried about and avoided unfamiliar situations, with concerns about what might happen and what might be expected of her. These worries led to considerable reassurance seeking from her parents and others around her and were associated with symptoms of muscular tension, impaired concentration and attention, fatigue, difficulty falling asleep, and irritability.

Finally, AR suffered from a long standing fear of the dark. AR was fearful of being attacked when outside at night and avoided being outside where possible.

History of presenting problem. AR presented with a long history of anxiety. Her mother described chronic worry, anxiety about being away from her mother, and shyness even as a young child. AR's anxiety intensified around 11 years of age when she commenced high school. She worried a great deal about the transition and found it particularly difficult to make new friends. In high school, AR was teased periodically, which served to intensify her social anxiety, and tendency to worry.

\section{Procedure and assessment measures}

The procedure was identical to that conducted with CF except that AR participated in BRAVE for Teenagers - ONLINE rather than the child program. Similarly, the assessment measures were the same as outlined for CF, except that AR also completed the Youth Self Report measure described above. At the time of writing, 6-month follow-up data were not yet available for AR as the teenage version of BRAVE - ONLINE is a very recent development and AR was one of the first participants.

\section{Results}

\section{Baseline assessment}

Table 2 summarizes AR's diagnostic information, CSR ratings, CGAS ratings and questionnaire measures at pre-treatment and post-treatment. According to the ADIS-C/P, AR was diagnosed with social phobia, GAD, specific phobia (natural environment type), and dysthymia. Social phobia was considered the primary diagnosis due to the marked impairment in social functioning, considerable avoidance and distress, and greater day-to-day impairment compared with her GAD and fear of the dark. Clinician-based assessment of AR's global functioning, as measured by the CGAS, placed AR within the clinical range.

As is evident from Table 2, results from the questionnaire measures were consistent with AR's diagnostic profile on the ADIS-C/P. AR scored within the clinical range on the social phobia and GAD subscales of both the SCAS-C and SCAS-P, as well as on the total score and panic/agoraphobia subscale of the SCAS-P. Her elevated scores on the panic/agoraphobia subscale of the SCAS-P reflected the mother's endorsement of items regarding anxious arousal and physiological symptoms in feared situations. AR scored within the clinical range for internalizing difficulties on the CBCL and in the borderline clinical range on the YSR. 
Table 2. Case Study 2 - Pre- to post-treatment and follow-up outcome scores and diagnostic information

\begin{tabular}{|c|c|c|}
\hline Measure & Pre & Post \\
\hline CSR Social phobia (Primary) & 6 & nil \\
\hline CSR GAD & 6 & nil \\
\hline CSR Specific phobia & 5 & nil \\
\hline CSR Dysthymia (provisional) & 4 & nil \\
\hline Combined CGAS & $39^{\# \#}$ & 71 \\
\hline \multicolumn{3}{|l|}{ SCAS-C } \\
\hline OCD & 2 & 2 \\
\hline Social phobia & $10^{\# \#}$ & $5^{*}$ \\
\hline Panic & 1 & 0 \\
\hline Separation & 5 & 2 \\
\hline Physical injury & 2 & 2 \\
\hline GAD & $9^{\# \#}$ & 6 \\
\hline Total & 29 & $15^{*}$ \\
\hline \multicolumn{3}{|l|}{ SCAS-P } \\
\hline OCD & 0 & 0 \\
\hline Social phobia & $12^{\# \#}$ & $6^{*}$ \\
\hline Panic & $6^{\# \#}$ & $0^{*}$ \\
\hline Separation & 3 & 2 \\
\hline Physical injury & 1 & 2 \\
\hline GAD & $7^{\# \#}$ & $5^{*}$ \\
\hline Total & $29^{\# \#}$ & $13^{*}$ \\
\hline \multirow{2}{*}{ CBCL - Internalizing } & $32^{\# \#}$ & 4 \\
\hline & $\mathrm{T}=77$ & $\mathrm{~T}=50^{*}$ \\
\hline \multirow[t]{2}{*}{ YSR - Internalizing } & $28^{\#}$ & 13 \\
\hline & $\mathrm{T}=67$ & $\mathrm{~T}=54^{*}$ \\
\hline Satisfaction - Parent & - & 40 \\
\hline Satisfaction - Child & - & 31 \\
\hline WAI & Session 3 & \\
\hline Child report & 79 & 67 \\
\hline Parent report & 84 & 74 \\
\hline
\end{tabular}

CSR = Clinician Severity Rating; CGAS = Children's Global Assessment Scale; SCAS-C = Spence Children's Anxiety Scale - Child Version; SCAS-P = Spence Children's Anxiety Scale - Parent Version; $\mathrm{CBCL}=$ Child Behaviour Checklist; YSR $=$ Youth Self Report ${ }^{\# \#}$ clinical range, " borderline clinical range, ${ }^{*} \mathrm{RCI}$ values indicate a reliable change at the $5 \%$ significance level or better

\section{Treatment participation}

Session and activity completion. Both AR and her mother completed most sessions efficiently. AR completed all in-session activities and approximately $85 \%$ of all homework tasks. Although session and activity completion was good, compliance with relaxation practice was poor. Despite therapist encouragement, AR practised relaxation on only 15 out of a possible 28 days. AR and her mother also demonstrated a 2-week delay in beginning graded exposure homework. 
Graded exposure tasks. AR designed her own exposure hierarchy, focusing on her primary diagnosis of social phobia, with the goal of being able to feel less anxious about talking to, and in front of, unfamiliar people. Initial steps included phoning a friend, ordering take-away food over the phone, initiating conversation with an unfamiliar person at college, asking a stranger for directions, and asking a shop assistant a question. Later steps focused on her fear of public speaking and included delivery of an oral presentation in front of family members, a friend, a group of friends and, finally, a group of college peers.

The online therapist noticed that AR had failed to complete the first two homework tasks for graded exposure. Email contact revealed that AR was feeling anxious about starting the exposure hierarchy. After the therapist emphasized the importance of exposure and encouraged AR to begin the first step, AR promptly completed the first step on her hierarchy. Thereafter, graded exposure proceeded well. During the 1-month booster session, the family reported excellent progress. AR had completed almost all hierarchy steps, and was feeling significantly more confident in social situations. Having experienced success with graded exposure, AR planned to develop another hierarchy designed to reduce her fear of the dark. Progress on this hierarchy will be evaluated during the 3-month booster session.

Self efficacy and treatment outcome expectancy. AR and her mother endorsed high levels of self efficacy in relation to the skills required for using the BRAVE - ONLINE treatment program (AR: 89/100; Mother: 97/100). Expectancy scores were also high (AR: 36/45; Mother: 41/45).

Therapeutic alliance. The WAI-S scores at Session 3 and post- treatment indicated a strong, positive, collaborative relationship between the therapist and AR and between the therapist and AR's mother, as shown in Table 2.

\section{Post-treatment results}

Clinical interviews revealed that AR no longer met diagnostic criteria for social phobia, GAD, specific phobia or dysthymia at post-treatment. AR's mother reported that her daughter no longer avoided social interactions, was considerably less anxious about talking to unfamiliar people, and was generally more confident and happy. AR reported that her self-esteem had improved, and that she was developing a stable peer group at college.

Interviewer ratings of AR's global functioning on the CGAS fell within the normal range at post-assessment, as did all subscales of the SCAS-C, most subscales of the SCAS-P, and the internalizing subscales of the YSR and CBCL. Only the GAD subscale of the SCAS-P remained in the clinical range.

The RCI calculations for the SCAS-C, SCAS-P, CBCL, and YSR indicated clinically reliable change for the social phobia subscales and the total scores of both the SCAS-P and SCAS-C, the panic subscale of the SCAS-P, and the internalizing subscales of both the CBCL and YSR.

Both AR and her mother reported high levels of consumer satisfaction with the program. They commented that the program had taught them both skills to manage anxiety more effectively and cope better with future anxiety-provoking situations. 


\section{Discussion}

Taken together, the outcomes for the case studies were generally positive and illustrate the ways in which an online CBT program can be used successfully in the treatment of child and adolescent anxiety. Both the young people and their parents engaged well in the online treatment, participating actively, and mostly complying with session schedules. Despite the predominantly online nature of therapist contact, an effective therapeutic alliance was able to be established in both cases.

Although treatment focused solely on the young person's primary diagnoses, significant reductions in anxiety were also evident for the secondary diagnoses, indicating that the CBT skills learned in relation to the primary diagnosis were generalized to other anxiety problems. The online mode of delivery was rated positively by the young people and their mothers, and the self-directed nature of the program was reported to be a key factor in their satisfaction.

Before conclusions can be drawn about the effectiveness of internet delivery of CBT for child and adolescent anxiety, we await the results of the randomized controlled trials that are currently underway. It will be particularly important to determine whether all families are sufficiently motivated to comply with and to complete the online program as conscientiously as the two cases reported here. The following section highlights some of the difficulties we have experienced to date in implementing the online program with other cases.

\section{Difficulties encountered with online therapy implementation}

\section{Exposure hierarchies}

One of the greatest challenges has been the design of appropriate exposure hierarchies. Despite inclusion of a phone call to assist with this process in the child program, and the addition of detailed guidelines in the adolescent program, the development of an appropriate hierarchy online, with minimal therapist contact, is difficult. With face-to-face contact it is much easier to obtain accurate details and ratings regarding feared situations, to ensure that the steps in exposure are appropriate, are not excessive and that the tasks are feasible. The design of appropriate hierarchies is a particular challenge where the child has multiple anxiety disorders, and other comorbid problems.

\section{Monitoring and problem solving}

Internet delivery of therapy produces some specific difficulties relating to monitoring and facilitating treatment progress. It is much harder for the therapist to explore factors such as treatment resistance and non-compliance over the internet compared to face-to-face contact. The reduced amount of verbal communication and absence of visual cues also reduces the amount of information available to the therapist.

\section{Delay in session completion}

Yet another difficulty that we are encountering with some families, particularly within the adolescent group, is failure to complete sessions on time. Online sessions become available one week after completion of the previous session. Despite automated email reminders to prompt completion, some families are consistently late in completing their sessions. Although 
online delivery has the advantage of flexibility for the family, it is also easy to put off completion of sessions when competing situations arise.

Panic disorder and agoraphobia, obsessive compulsive disorder and posttraumatic stress disorder

The original program focused primarily on the treatment of separation anxiety, social phobia, generalized anxiety disorder and simple phobias. Many of the children and adolescents referred to the program exhibit other comorbid anxiety disorders, such as panic disorder and agoraphobia, obsessive compulsive disorder, and posttraumatic stress disorder and are not accepted for treatment. Although the core material of the program is common to the treatment of all anxiety disorders, there is a need for adjunct modules that provide additional therapy with content specific to these presenting problems.

\section{Suggestions for future research}

Computer-based therapy is an exciting innovation, with numerous avenues for further investigation. One direction will be to investigate whether the internet treatment is feasible and effective if used as a self-help intervention, with no direct assistance from a therapist. From a public health perspective even a small effect size can be of major importance in a large population, and it will be valuable to conduct a cost-benefit analysis comparing self-help/online, therapist assisted/on-line and clinic delivery of the intervention.

We also intend to examine the effectiveness of the program when used by school counsellors, as an adjunct to brief consultations. The self-help and counsellor-assisted versions could also be used in the context of prevention of anxiety disorders for students at risk.

Another avenue for future research involves exploring the importance of parental involvement in online treatment. There may be differences in the value of parent involvement depending upon the age of the child, or an optimal level of parental involvement at different ages. The development and evaluation of additional strategies to enhance the therapeutic alliance and client motivation are also areas for future research. For example, we intend to investigate the impact of rewards on treatment compliance.

\section{Conclusion}

In conclusion, the present paper illustrates the feasibility of translating CBT for child and adolescent anxiety into online delivery. The conversion from a traditional face-to-face, clinicbased approach presented a number of challenges, particularly in relation to methods for enhancement of the therapist-client relationship and maximization of client motivation. The paper described many of the techniques used to overcome these challenges, but also illustrated that there are still numerous complexities involved. The two case studies suggest that online CBT can be effective in treating youth anxiety. However, we cannot yet draw conclusions about the general efficacy and effectiveness of this approach until data are available from large scale randomized controlled trials. The paper identified many directions for future research, and tentatively proposes that therapist assisted, online CBT offers promise in the treatment of child and adolescent anxiety disorders. 


\section{Acknowledgements}

We acknowledge the National Health and Medical Research Council of Australia for funding this project and Shockmedia, our web designers, without whom BRAVE - ONLINE would not have become a reality.

\section{References}

Achenbach, T. M. and Rescorla, L. A. (2001). Manual for the ASEBA School-Age Forms and Profiles. Burlington, VT: University of Vermont, Research Centre for Children, Youth and Families.

Bandura, A. (1977). Social Learning Theory. Englewood Cliffs, NJ: Prentice-Hall.

Barrett, P. M. (1998). Evaluation of cognitive-behavioral group treatments for childhood anxiety disorders. Journal of Clinical Child Psychology, 27, 459-468.

Booth, M. L., Bernard, D., Quine, S., Kang, M. S., Usherwood, T., Alperstein, G. and Bennett, D. L. (2004). Access to health care among Australian adolescents: young people's perspectives and their sociodemographic distribution. Journal of Adolescent Health, 34, 97-103.

Borkovec, T. D. and Nau, S. D. (1972). Credibility of analogue therapy rationales. Journal of Behavior Therapy and Experimental Psychiatry, 3, 257-260.

Cornwall, E., Spence, S. H. and Schotte, D. (1996). The effectiveness of emotive imagery in the treatment of darkness phobia in children. Behaviour Change, 13, 223-229.

Dadds, M. R., Davey, G. C. L. and Field, A. P. (2001). Developmental aspects of conditioning processes in anxiety disorders. In M. W. Vasey and M. R. Dadds (Eds.), The Developmental Psychopathology of Anxiety. Oxford: Oxford University Press.

Dewis, L. M., Kirkby, K. C., Martin, F., Daniels, B. A., Gilroy, L. J. and Menzies, R. G. (2001). Computer-aided vicarious exposure versus live graded exposure for spider phobia in children. Journal of Behavior Therapy and Experimental Psychiatry, 32, 17-27.

Essau, C. A., Conradt, J. and Petermann, F. (2000). Frequency, comorbidity, and psychosocial impairment of anxiety disorders in German adolescents. Journal of Anxiety Disorders, 14, 263279.

Fung, D. S. S., Manassis, K., Kenny, A. and Fiksenbaum, L. (2002). Web-based CBT for selective mutism. Journal of the American Academy of Child and Adolescent Psychiatry, 41, 112113.

Griffiths, K. M. and Christensen, H. (2006). Review of randomised controlled trials of internet interventions for mental disorders and related conditions. Clinical Psychologist, 10, 16-29.

Howgego, I. M., Yellowlees, P., Owen, C., Meldrum, L. and Dark, F. (2003). The therapeutic alliance: the key to effective patient outcome? A descriptive review of the evidence in community mental health case management. Australian and New Zealand Journal of Psychiatry, 37, 169-183.

Hudson, J. L. (2005). Efficacy of cognitive-behavioural therapy for children and adolescents with anxiety disorders. Behaviour Change, 22, 55-70.

Iverson, G. L. (2001). Interpreting change on the WAIS-III/WMS-III in clinical samples. Archives of Clinical Neuropsychology, 16, 183-191.

Jacobson, N. S. and Truax, P. (1991). Clinical significance: a statistical approach to defining meaningful change in psychotherapy research. Journal of Consulting and Clinical Psychology, 59, 12-19.

Kaltenthaler, E., Brazier, J., De Nigris, E., Tumur, I., Ferriter, M., Berverley, C., Parry, G., Rooney, G. and Sutcliffe, P. (2006). Computerised cognitive behaviour therapy for depression and anxiety update: a systematic review and economic evaluation. Health Technology Assessment, 10, $1-186$.

Kenardy, J. A. and Adams, C. (1993). Computers in cognitive-behaviour therapy. Australian Psychologist, 28, 189-194. 
Kenardy, J. A., Dow, M. G. T., Johnston, D. W., Newman, M. G., Thomson, A. and Taylor, C. B. (2003). A comparison of delivery methods of cognitive-behavioral therapy for panic disorder: an international multicenter trial. Journal of Consulting and Clinical Psychology, 71, 1068-1075.

Kendall, P. C. (1994). Treating anxiety disorders in children: results of a randomized clinical trial. Journal of Consulting and Clinical Psychology, 62, 100-110.

Lyneham, H. J. and Rapee, R. M. (2005). Agreement between telephone and in-person delivery of a structured interview for anxiety disorders in children. Journal of the American Academy of Child and Adolescent Psychiatry, 44, 274-282.

Martin, D. J., Garske, J. P. and Davis, M. K. (2000). Relation of the therapeutic alliance with outcome and other variables: a meta-analytic review. Journal of Consulting and Clinical Psychology, 68, 438-450.

Muris, P., Schmidt, H. and Merckelbach, H. (2000). Correlations among two self-report questionnaires for measuring DSM-defined anxiety disorder symptoms in children: the Screen for Child Anxiety Related Emotional Disorders and the Spence Children's Anxiety Scale. Personality and Individual Differences, 28, 333-346.

Nauta, M. H., Scholing, A., Rapee, R. M., Abbott, M., Spence, S. H. and Waters, A. (2004). A parentreport measure of children's anxiety: Psychometric properties and comparison with child-report in a clinic and normal sample. Behaviour Research and Therapy, 42, 813-839.

Nelissen, I., Muris, P. and Merckelbach, H. (1995). Computerized exposure and in vivo exposure treatments of spider fear in children: two case reports. Journal of Behavior Therapy and Experimental Psychiatry, 26, 153-156.

Ollendick, T. H., King, N. J. and Muris, P. (2002). Fears and phobias in children: phenomenology, epidemiology, and aetiology. Child and Adolescent Mental Health, 7, 98-106.

Rapee, R. M. and Spence, S. H. (2004). The etiology of social phobia: empirical evidence and an initial model. Clinical Psychology Review. Special Social Phobia and Social Anxiety, 24, 737-767.

Rapee, R. M., Wignall, A., Hudson, J. L. and Schniering, C. A. (2000). Treating Anxious Children and Adolescents: an evidence-based approach. Oakland, CA: Harbinger Publications.

Schaffer, D., Gould, M. S., Brasic, J., Ambrosini, P., Fisher, D., Bird, H. and Aluwahlia, S. (1983). A Children's Global Assessment Scale (CGAS). Archives of General Psychiatry, 40, 1228-1231.

Silverman, W. K. (1994). Structured diagnostic interviews. In T. H. Ollendick and N. J. King (Eds.), International Handbook of Phobic and Anxiety Disorders in Children and Adolescents. issues in clinical child psychology (pp. 293-315). New York: Plenum Press.

Silverman, W. K. and Albano, A. M. (1996). Anxiety Disorders Interview schedule for DSM-IV-Child Version: Parent Interview Schedule. San Antonio: The Psychological Corporation - Harcourt, Brace and Company.

Silverman, W. K. and Treffers, P. D. A. (2001). Anxiety Disorders in Children and Adolescents: research, assessment and intervention. New York: Cambridge University Press.

Spek, V., Cuijpers, P., NyklÃcek, I., Riper, H., Keyzer, J. and Pop, V. (2007). Internet-based cognitive behaviour therapy for symptoms of depression and anxiety: a meta-analysis. Psychological Medicine, 37, 319-328.

Spence, S. H. (1998). A measure of anxiety symptoms among children. Behaviour Research and Therapy, $36,545-566$.

Spence, S. H., Barrett, P. M. and Turner, C. M. (2003). Psychometric properties of the Spence children's anxiety scale with young adolescents. Journal of Anxiety Disorders, 17, 605-625.

Spence, S. H., Donovan, C. and Brechman-Toussaint, M. (2000). The treatment of childhood social phobia: the effectiveness of a social skills training-based, cognitive-behavioural intervention, with and without parental involvement. Journal of Child Psychology and Psychiatry, 41, 713-726.

Spence, S. H., Holmes, J. M., Donovan, C. L. and Kenardy, J. (2006). BRAVE for Teenagers ONLINE: an internet based program for adolescents with anxiety. Brisbane, Australia: School of Psychology, University of Queensland. 
Spence, S. H., Holmes, J. M., March, S. and Lipp, O. V. (2006). The feasibility and outcome of clinic plus internet delivery of cognitive-behavior therapy for childhood anxiety. Journal of Consulting and Clinical Psychology, 74, 614-621.

Spence, S. H., March, S. and Holmes, J. M. (2005). BRAVE for Children - ONLINE: an internet based program for children with anxiety. Brisbane, Australia: School of Psychology, University of Queensland.

Tracey, T. J. and Kokotovic, A. M. (1989). Factor structure of the Working Alliance Inventory. Psychological Assessment, 1, 207-210.

Vasey, M. W. and Dadds, M. R. (2001). The Developmental Psychopathology of Anxiety. London: Oxford University Press. 